\title{
Stress-strain Hysteresis of a Carbon Nanotube Network as Polymer Nanocomposite Filler under Cyclic Deformation
}

\author{
Petr Slobodian and Petr Saha \\ Centre of Polymer Systems, Polymer Centre, Tomas Bata University in Zlin, \\ nam. T. G. Masaryka 5555, 76001 Zlin, Czech Republic
}

\begin{abstract}
A network of entangled multiwall carbon nanotubes is an assembly of non-bonded and randomly oriented units whose mechanical behavior is affected by their buckling, compression and slippage at contact points. Slippage and irreversible nanotube rearrangements are evidenced by the hysteresis in the stress-strain curves during loading and unloading cycles. To model the mechanical behavior, a nonlinear rheological model is proposed as a combination of the elastic and the friction stresses and a kinetic equation.
\end{abstract}

Keywords: Carbon nanotubes, Entangled network, Mechanical properties, Cyclic deformation. PACS: 81.05Qk, 81.07De, 83.60Df

\section{INTRODUCTION}

Recent technology progress relies heavily on the use of materials that can offer advanced structural and functional capabilities. In this respect, entangled carbon nanotube network structures of buckypaper show a great potential for developing high-performance polymer composite materials. The network can proportionally transfer its unique properties into composites and bring substantial improvements in structural strength, electrical and thermal conductivity, electromagnetic interference shielding and other properties in comparison to polymer composites with carbon nanotube particulate filling $[1,2]$. However, incorporation of conductive nanotube network into a hosting polymer matrix is a difficult task. Traditionally, the network is fixed by a polymer solution (epoxy or bismaleimide resin, polycarbonate solution) to form polymer composites [3, 4].

The abovementioned manufacturing of nanotube network based polymer composite is rather laborious and may be circumvent by interlocking porous filtrating membrane with entangled nanotubes. The novel process consists of using the flexible non-woven polystyrene (PS) filter as supporting and integrating element at which, in our case, the multiwall carbon nanotubes (MWCNT) settle and form a network during MWCNT suspension filtration [5-7]. The obtained MWCNT/PS composite can be used either without adjustment or hot compressed to increase MWCNT fixing to PS membrane. 
The repeated layering of MWNT/PS membrane sheets yields bulky forms. The processing seems promising for a continuous manufacture of carbon nanotube network/polymer composites since the filter-support ensures itself the composite compactness. The usual peeling off the MWCNT network from the membrane is eliminated as well as the network impregnation by means of polymer solutions to increase its mechanical integrity.

The aim of this paper is to study the stress-strain dependence of MWCNT network. A recent study investigates the mechanical behavior of entangled mats of carbon nanotubes and several other fibers during compression and cyclic tests [8]. The obtained hysteresis loop between loading and unloading was linked with mat morphology and motion, friction and rearrangement of fibers during compaction. We investigate this issue with our MWCNT network to determine a steady-state cyclic regime. This regime is important for the use of the network as pressure sensing elements.

\section{EXPERIMENTAL}

Purified MWCNT of acetylene type were supplied by Sun Nanotech Co. Ltd., China. According to the supplier, the nanotube diameter is $10-30 \mathrm{~nm}$, length $1-10 \mu \mathrm{m}$, purity $>90 \%$ and volume resistance $0.12 \Omega . \mathrm{cm}$. The nanotubes were used for the preparation of aqueous paste: $1.6 \mathrm{~g}$ of MWCNT and $\sim 50 \mathrm{ml}$ of deionized water were mixed with the help of a mortar and pestle. The paste was then diluted in deionized water with sodium dodecyl sulfate (SDS) and 1-pentanol. Consequently, $\mathrm{NaOH}$ aqueous solution was added to adjust $\mathrm{pH}$ to the value of 10 . The final nanotube concentration in the suspension was $0.3 \mathrm{wt}$. \%, concentration of SDS and 1-pentanol $0.1 \mathrm{M}$ and $0.14 \mathrm{M}$, respectively. The suspension was sonicated in Dr. Hielscher GmbH apparatus (ultrasonic horn S7, amplitude $88 \mu \mathrm{m}$, power density $300 \mathrm{~W} . \mathrm{cm}^{-2}$, frequency $24 \mathrm{kHz}$ ) for 2 hours and the temperature of ca $50^{\circ} \mathrm{C}$.

For making entangled MWCNT network on a polyurethane porous membrane [9], a vacuum filtration method was used. The formed disk-shaped network was washed several times by deionized water and methanol in situ, then removed and dried between filter papers. The thickness of the obtained disks was typically $0.15-0.46 \mathrm{~mm}$ (Figure 1a).

The structure of MWCNT network was investigated with a scanning electron microscope (SEM) made by Vega Easy Probe (Tescan s.r.o., Czech Republic). The sample taken from the disk was first deposited onto carbon targets and covered with a thin $\mathrm{Au} / \mathrm{Pd}$ layer. For the observations the regime of secondary electrons was chosen.

The MWCNT/PS composite is prepared by flow filtration of aqueous MWCNT dispersion through polystyrene (PS) filtrating membrane. The start-up phase when the nanotubes are infiltrated into the PS filter mesh is followed by sedimentation of MWCNT. PS filter-supported filtrate is several times washed by deionized water and methanol in situ. Then the composite is placed between filter papers moisten in acetone and dried between two iron plates at the room temperature for one day. The final drying continued without iron plates at $40^{\circ} \mathrm{C}$ throughout another day. The 
thickness of the non-woven PS filter is typically $0.5 \mathrm{~mm}$ and the MWCNT entangled network 0.02-0.4 mm. PS filter-supported MWCNT network composite can be used either without adjustment or the hot compression molding at $190^{\circ} \mathrm{C}$ converts PS fiber membrane to a film with fixed MWCNT network.

The PS membrane is prepared by electrospinning from solution. PS is solved in a mixture of methyl isobutyl ketone and dimethyl formamide with the volume ratio 3:1 (PS weight concentration is 15 wt. \%). The nanofiber layer is made using NanoSpider (Elmarco, s.r.o.) equipped by the steel rotation electrode with needles and the steel cylinder collecting electrode (details in [9]). Then the porous layer is subjected to hot pressing at pressure $0.6 \mathrm{MPa}$ and temperature $80^{\circ} \mathrm{C}$.

The MWCNT networks were tested for deformation using a simple set-up. The network stripe (length $10 \mathrm{~mm}$ and width $8 \mathrm{~mm}$, the loading area between glass plates was $8 \times 8 \mathrm{~mm}$ ) cut out from the manufactured disks of entangled CNT was stepwise compressed between two glasses to the maximum value with $20 \mathrm{~s}$ delay of strain reading in each step. Then the down-stress curve was measured in the same manner.

\section{RESULTS}

The structure of the upper surface of entangled nanotubes can be seen in Figure $1 \mathrm{~b}$ as SEM micrograph. After filtration of MWCNT suspension the network was dried, which caused its shrinkage by about 7\%. The porosity was calculated from relation $\phi=1-\rho_{\text {net }} / \rho_{\text {MWCNT }}$, where $\rho_{\text {net }}=0.56 \pm 0.03 \mathrm{~g} \cdot \mathrm{cm}^{-3}$ denotes the measured apparent density of the nanotube network. The calculated porosity $\phi=0.67$ corresponds well to the published values for MWCNT networks [10].

Mechanical properties of manufactured structures were followed in the course of twelve compression and relaxation cycles with cyclic accumulation of residual strain. The measured stress and strain absolute values are in the form of compressive stress vs. strain dependence presented in Figure 2.

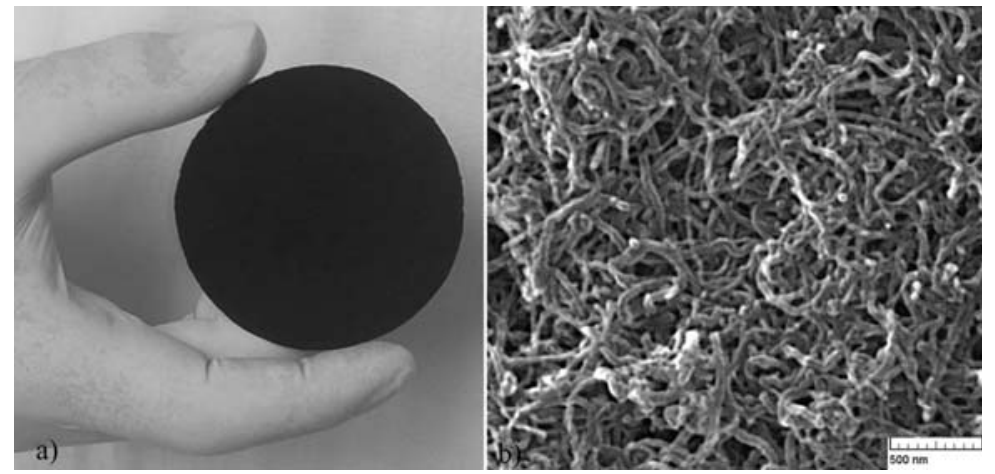

FIGURE 1. Free-standing randomly entangled MWCNT network (disk diameter $75 \mathrm{~mm}$, thickness $0.15 \mathrm{~mm}$ ) (left), and SEM image of the surface of entangled MWCNT network. 

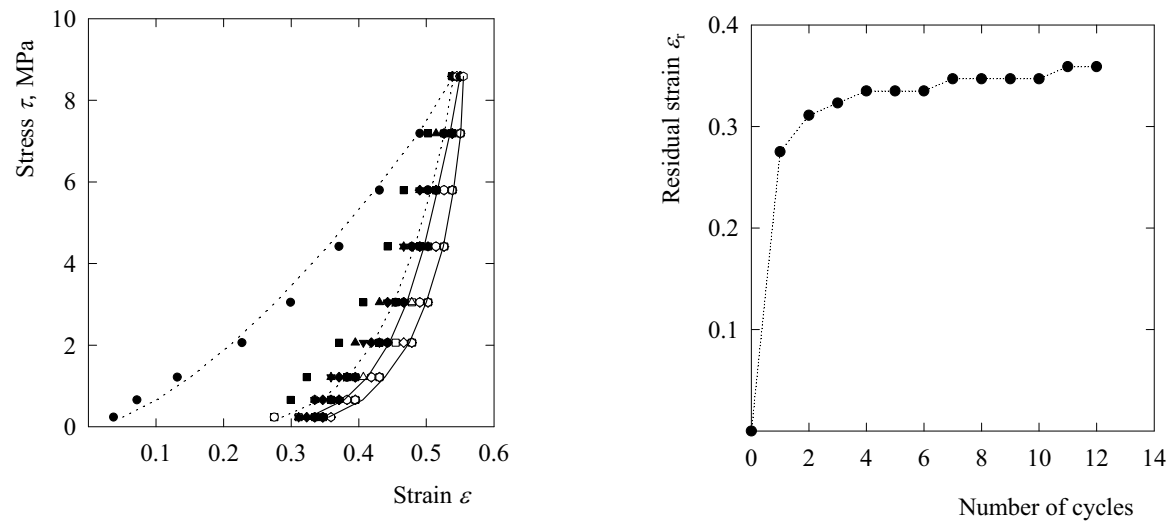

FIGURE 2. (Left) Absolute stress-strain loops in cyclic compression test for MWCNT network subjected to 12 compression/expansion cycles (the network thickness $0.418 \mathrm{~mm}$ ). The dotted lines (first loading and unloading cycle) and solid lines (twelfth cycle) represent the prediction given by Eq. 6 .

(Right) The plot of absolute residual strain vs. number of cycles.

The accumulation of residual strain is often called ratcheting and the minimum strain in each cycle is defined as ratcheting strain, $\varepsilon_{\mathrm{r}}$. In MWCNT network a ratcheting strain appears after the first compression cycle, probably due to the initial deformation of porous structure and blocked reverse motion of nanotubes inside the compact network, as hypothesized in [8]. Thus the ability of the network to be repeatedly highly compressed is reduced. Moreover, during successive cycles of loading and unloading the ratcheting rate per cycle decreases and an asymptotic value of $\varepsilon_{\mathrm{r}}$ is obtained, as demonstrated in Figure 3.

\section{Model of Cyclic Deformation}

The filtering type of MWCNT network is an assembly of entangled, non-bonded and randomly oriented carbon nanotubes. When the compressive load is applied, the common opinion on deformation micromechanics of wad assembly mostly emphasizes the fiber buckling, compression, contact points formation, slippage with friction at contact points and irreversible fiber rearrangement reflected in the hysteresis stressstrain curves during loading and unloading cycles (Figure 2), [11, 12]. However, the existing micromechanics models which relate the compressive stress and deformation of the randomly oriented mats $[13,14]$ are based on the bending deformation of fibers without frictional slippage at contact points. This simplifying assumption involves the affine motion of contacts, that is, the motion follows the macroscopic strain of the network [13]. Nevertheless, the aspect specific to non-bonded entangled networks is unit slippage at contact points which strongly depends in friction between units [12].

To generalize the bending assumption restricted to bonded assembly, we propose for non-bonded nonotube networks that the total compressive stress is a combination of the elastic bending stress and the friction stress 


$$
\sigma=\sigma_{e}+\sigma_{f}
$$

The micromechanical analyses of fiber assembly led to power law dependence of the bending stress to the fiber volume fraction with the power law index $n$ equal to 3 for random 3D structure [13, 14] and 5 for a planar network structure [14]. This dependence in terms of strain has the form

$$
\sigma_{b}=k_{E}\left(\varepsilon^{n}-\varepsilon_{r}^{n}\right)
$$

where $\varepsilon$ denotes the compressive (engineering) strain, $\varepsilon_{r}$ the residual compressive strain, $k_{E}=k E$, where $E$ denotes the elastic modulus of nanotubes and $k$ an adjustable parameter of the model which accounts for nanotube orientation distribution, the degree of waviness and loading direction.

The friction stress is proportional to the contact force. The contact force increases with compressive nanotube packing. Consequently, the friction stress is assumed to follow proportionally the compressive strain

$$
\sigma_{f}=\mu\left(\varepsilon-\varepsilon_{r}\right)
$$

where $\mu$ denotes the friction coefficient.

The hysteresis loops in Figure 2 show that the compressive stress increases with rising rate in the course of loading period. On the other hand, the compression decreases abruptly at the beginning of release. To include the possible mechanism into the stress-strain relation, Eq. 1, a scalar structural parameter $\lambda$ is used to characterize the instantaneous network structure. A value of unity may reflect the network deformation owing to slippage at the contact points. A decreasing $\lambda$ value may correspond to increasing bending deformation of nanotubes owing to their tightened contacts by the compression. The progress of the structural change is expressed by the first-order kinetic equation as

$$
d \lambda / d \varepsilon=-\lambda \quad, \lambda=\exp (-\varepsilon)
$$

Thus the structural parameter determines the relative importance of the friction at contact points with deformation progress as

$$
\sigma_{f}=\mu\left(\varepsilon-\varepsilon_{r}\right)\left\{\exp \left[-\left(\varepsilon-\varepsilon_{r}\right)\right]\right\} .
$$

At the same time, the compression of contacts prevents nanotube slippage and the bending deformation takes place

$$
\sigma_{e}=k_{E}\left(\varepsilon^{n}-\varepsilon_{r}^{n}\right)\left\{1-\exp \left[-\left(\varepsilon-\varepsilon_{r}\right)\right]\right\} .
$$



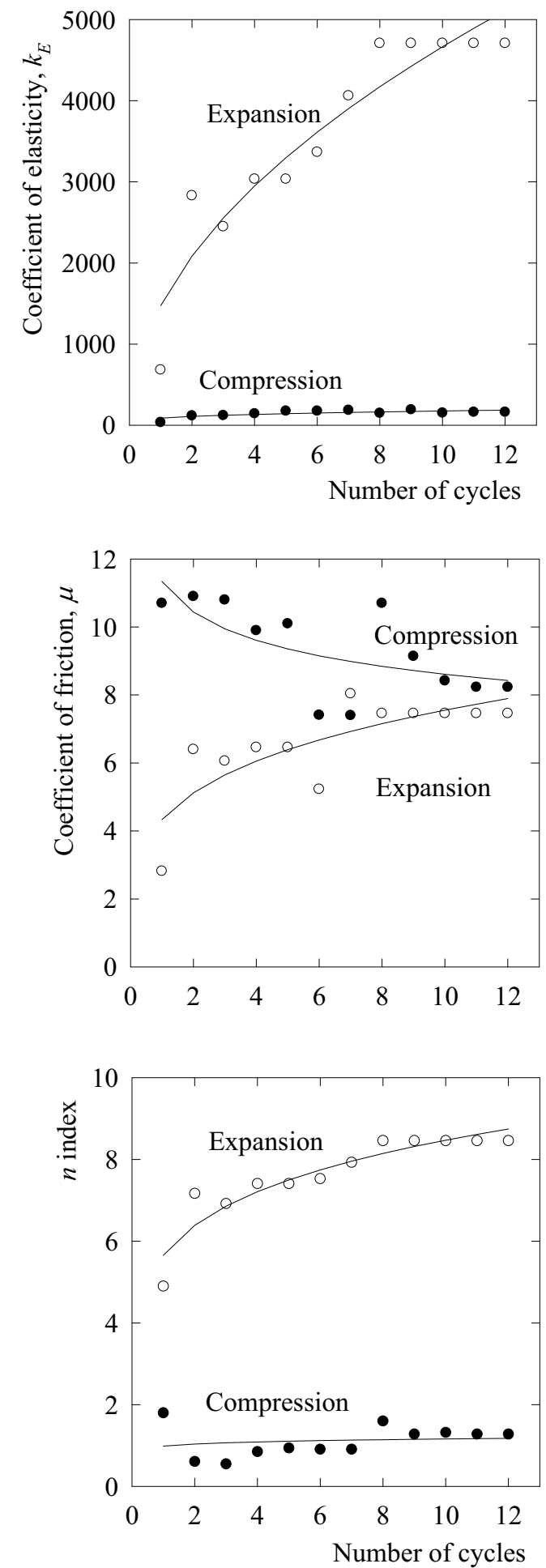

FIGURE 3. The cycle dependence of parameters in Eqs. 5 and 6 for deformation of nanotube network in compression/expansion cycles presented in Figure 2. The symbols denote calculated values and the lines the power law representation. 
The parameter values for the stress vs. strain relation (1), (5) and (6) presented in Figure 3 suggest the possible significance of bending and friction motion of nanotubes in the course of network deformation in compression/expansion cycles. The friction term, Eq. 5, approaches to the asymptotic value with increasing number of cycles which is equivalent both for compression and expansion. The effect of friction is stabilized by the cycles and thus reversible. On the other hand, the elasticity parameter $k_{E}$ and the power law index $n$ are considerably higher for the unloading period than for loading one. Accordingly to the proposed description of the network deformation, the bending term prevails significantly the friction term in the course of network expansion. It suggests that the deformed and compact network is deformed mainly due to nanotube bending while the network compression in the initial cycles is linked with the nanotube slippage at the contact points.

\section{CONCLUSION}

The stress/strain characteristics for entangled CNT network structures of buckypaper produced by filtering a nanotube suspension have not been studied in details so far. Thus the primary aim of our research was to find out the effect of compression on network deformation when a repeated loading is exerted. The measurements have shown hysteresis loading/unloading loops and the residual network deformation. The structure recovering mechanism projects into the residual strain, the change of which decreases with the increasing number of applied cycles and finally, an asymptotic value of the residual strain is reached. During successive cycles of loading and unloading the nanotubes rearrangement becomes steady and MWCNT network reaches also a stable stress-strain hysteresis loop shape. It means that the entangled carbon nanotube network structure can be used as a sensing element of compressive stress, especially when the network is suitably deformed in advance. The asymptotic value of residual strain manifests the irreversible fiber rearrangement after the repeated deformation cycles and stable hysteresis stress-strain curves.

The proposed mechanism of network deformation assumes the combination of the nanotube bending and the slippage with friction at contact points. According to this concept, the compressed network is deformed mainly due to nanotube bending while the network deformation in the initial cycles is linked with the nanotube slippage at the contact points.

\section{ACKNOWLEDGMENTS}

This article was created with support of Operational Programme Research and Development for Innovations co-funded by the European Regional Development Fund (ERDF) and national budget of Czech Republic within the framework of the Centre of Polymer Systems project (reg.number: CZ.1.05/2.1.00/03.0111). 


\section{REFERENCES}

1. E. T. Thostenson, C. Y. Li and T. W. Chou, Comp. Sci. Techn. 65, 491-516 (2005).

2. Q. Cao and J. A. Rogers, Adv. Mat. 21, 29-53 (2009).

3. Z. Wang, Z. Liang, B. Wang, C. Zhang and L. Kramer, Composites: Part A 35, 1225-1232 (2004).

4. T. W. Chou, L. Gao, E. T. Thostenson, Z. Zhang and J. H. Byun, Comp. Sci. Techn. 70, 1-19 (2010).

5. P. Slobodian, P. Riha, A. Lengalova and P. Saha, J. Expl. Nanoscience (2011). (In Press).

6. P. Slobodian, P. Riha, A. Lengalova and P. Saha, J. Mater. Sci. (2011). DOI: 10.1007/s10853-0105202-0.

7. P. Slobodian, P. Riha, R. Olejnik, D. Petras, M. Machovsky and P. Saha, Measurement Sci. Techn. (2011). (In Press).

8. D. Poquillon, B. Viguier and E. Andrieu, J. Mat. Sci. 40, 5963-5970 (2005).

9. D. Kimmer, P. Slobodian, D. Petras, M. Zatloukal, R. Olejník and P. Saha, J. Appl. Polym. Sci. 111, 2711-2714 (2009).

10. R. L. D. Whitby, T. Fukuda, T. Maekawa, S. L. James and S. V. Mikhalovsky, Carbon 46, 949-956 (2008).

11. S. B. Stankovic, Polym. Eng. Sci. 48, 676-682 (2008).

12. C. Barbier, R. Dendievel and D. Rodney, Phys. Rev. E 80016115 (2009).

13. C. M. van Wyk, J. Textile Inst. 37, T285-T292 (1946).

14. S. Toll, Polym. Eng. Sci. 38, 1337-1350 (1998). 
Copyright of AIP Conference Proceedings is the property of American Institute of Physics and its content may not be copied or emailed to multiple sites or posted to a listserv without the copyright holder's express written permission. However, users may print, download, or email articles for individual use. 